\title{
Does the Jet Production Efficiency of Radio Galaxies COntrol Their Optical AGN TyPes? ${ }^{\dagger}$
}

\author{
SAScha TRIPPE \\ Department of Physics and Astronomy, Seoul National University, 599 Gwanak-ro, Gwanak-gu, Seoul 151-742, Korea \\ trippe@astro.snu.ac.kr
}

Received August 11, 2014; accepted August 22, 2014

\begin{abstract}
The jet production efficiency of radio galaxies can be quantified by comparison of their kinetic jet powers $P_{\text {jet }}$ and Bondi accretion powers $P_{\mathrm{B}}$. These two parameters are known to be related linearly, with the jet power resulting from the Bondi power by multiplication with an efficiency factor of order $1 \%$. Using a recently published (Nemmen \& Tchekhovskoy 2014) high-quality sample of 27 radio galaxies, I construct a $P_{\mathrm{B}}-P_{\text {jet }}$ diagram that includes information on optical AGN types as far as available. This diagram indicates that the jet production efficiency is a function of AGN type: Seyfert 2 galaxies seem to be systematically (with a false alarm probability of $4.3 \times 10^{-4}$ ) less efficient, by about one order of magnitude, in powering jets than Seyfert 1 galaxies, LINERs, or the remaining radio galaxies. This suggests an evolutionary sequence from Sy 2s to Sy 1s and LINERs, controlled by an interplay of jets on the one hand and dust and gas in galactic nuclei on the other hand. When taking this effect into account, the $P_{\mathrm{B}}-P_{\text {jet }}$ relation is probably much tighter intrinsically than currently assumed.
\end{abstract}

Key words: galaxies: active; jets; nuclei; Seyfert — accretion, accretion disks

\section{INTRODUCTION}

Active galactic nuclei (AGN; see, e.g., Netzer 2013 for a recent review) are supposedly powered by accretion of gas onto supermassive black holes located in the centers of probably all galaxies. A frequent, albeit as yet poorly understood, consequence of this process is the formation of collimated outflows, jets, which are emitters of synchrotron radiation especially prominent at radio wavelengths (see, e.g., Böttcher, Harris, \& Krawczynski 2012 for a recent review). One road to a better understanding of jet formation and the interplay of jets and accretion flows is provided by analysis of the energetics of the jets of nearby $(\lesssim 100 \mathrm{Mpc})$ radio galaxies which have accretion rates much smaller $(\ll 1 \%)$ than their Eddington limits. X-ray observations of those systems provide independent estimates of (i) the kinetic jet powers $P_{\text {jet }}$ and (ii) the Bondi accretion powers $P_{\mathrm{B}}=\dot{M}_{\mathrm{B}} c^{2}$, with $\dot{M}_{\mathrm{B}}$ being the Bondi accretion rate and $c$ denoting the speed of light. Multiple studies (Allen et al. 2006; Balmaverde, Baldi, \& Capetti 2008; Russell et al. 2013; King et al. 2013; Nemmen \& Tchekhovskoy 2014; Fujita, Kawakatu, \& Shlosman 2014) concluded that, within errors, $P_{\text {jet }}$ is proportional to $P_{\mathrm{B}}$. Accordingly, one finds a jet production efficiency $\eta$ from $P_{\text {jet }}=\eta P_{\mathrm{B}}$; observationally, $\eta \sim 1 \%$ (ensemble average). However, the observed $P_{\mathrm{B}}-P_{\text {jet }}$ relations show substantial ( $\gtrsim 0.5 \mathrm{dex}$ ) intrinsic scatter, indicating an as yet overlooked systematic effect. This article addresses a possible cause.

Corresponding author: S. Trippe

$\dagger$ RAPid COMMUNiCATion

\section{DAta AND ANAlysis}

I make use of the data set published recently by Nemmen \& Tchekhovskoy (2014) [their Table 1] which has in turn been drawn from data by Balmaverde, Baldi, \& Capetti (2008) and Russell et al. (2013) and which is marked by very careful selection. Jet and Bondi powers were derived from X-ray observations obtained by the Chandra space telescope. Kinetic jet powers were estimated from the ages and creation energies of jetinflated X-ray cavities. Bondi accretion rates were estimated from temperature and density profiles of hot diffuse gas. (Please refer to Nemmen \& Tchekhovskoy 2014 for technical details.)

I collected optical AGN types from the NASA/IPAC Extragalactic Database (NED); the SIMBAD Astronomical Database; Ho , Filippenko, \& Sargent (1997); and Véron-Cetty \& Véron (2010). Eventually, I found optical types for 17 of my 27 targets: two are of type Seyfert 1, six of type Seyfert 2, and nine of type Low-Ionization Nuclear Emission-Line Region galaxy (LINER - cf., Ho 2008). The final dataset is summarized in Table 1.

From the data of Table 1, I constructed a logarithmic $P_{\mathrm{B}}-P_{\text {jet }}$ diagram (Figure 1 ) that includes the available information on optical AGN types. The sample spans about three orders of magnitude in both jet power and accretion power. I determined the best-fit linear relation between jet and accretion power via a least-squares fit, leading to a (ensemble averaged) jet production efficiency of $\eta \approx 0.6 \%$ for my sample. The intrinsic scatter (i.e., the difference in squares of r.m.s. residual and bivariate r.m.s. measurement error) is about 0.5 dex. 
Table 1

The radio galaxy sample.

\begin{tabular}{|c|c|c|c|}
\hline Galaxy & Type & $\log P_{\mathrm{B}}[\mathrm{W}]$ & $\log P_{\text {jet }}[\mathrm{W}]$ \\
\hline $3 \mathrm{C} 066 \mathrm{~B}$ & Sy 1 & $38.59_{-0.16}^{+0.12}$ & $37.25 \pm 0.40$ \\
\hline 3C 083.1 & & $39.11_{-0.13}^{+0.10}$ & $36.69 \pm 0.40$ \\
\hline $3 \mathrm{C} 270$ & L 2 & $38.41_{-0.16}^{+0.12}$ & $36.77 \pm 0.40$ \\
\hline 3C 296 & & $39.36_{-0.20}^{+0.14}$ & $37.05 \pm 0.40$ \\
\hline 3C 449 & & $38.48_{-0.21}^{+0.14}$ & $36.66 \pm 0.40$ \\
\hline $3 \mathrm{C} 465$ & Sy 1 & $39.15_{-0.12}^{+0.10}$ & $37.58 \pm 0.40$ \\
\hline UGC 6297 & Sy 2 & $36.68_{-0.31}^{+0.18}$ & $34.83 \pm 0.40$ \\
\hline UGC 7386 & $\mathrm{~L} 1$ & $37.95_{-0.24}^{+0.16}$ & $36.18 \pm 0.40$ \\
\hline UGC 7898 & & $37.81_{-0.12}^{+0.09}$ & $35.53 \pm 0.40$ \\
\hline UGC 8745 & Sy 2 & $38.62_{-0.26}^{+0.16}$ & $35.78 \pm 0.40$ \\
\hline NGC 1399 & Sy 2 & $38.45_{-0.14}^{+0.11}$ & $35.34 \pm 0.40$ \\
\hline NGC 3557 & & $38.02_{-0.23}^{+0.15}$ & $35.87 \pm 0.40$ \\
\hline IC 1459 & $\mathrm{~L}$ & $38.48_{-0.12}^{+0.09}$ & $36.89 \pm 0.40$ \\
\hline IC 4296 & $\mathrm{~L}$ & $39.23_{-0.10}^{+0.08}$ & $36.94 \pm 0.40$ \\
\hline NGC 6166 & & $37.60_{-0.30}^{+0.18}$ & $35.95_{-0.11}^{+0.16}$ \\
\hline NGC 4696 & $\mathrm{~L}$ & $36.71_{-0.05}^{+0.05}$ & $36.15_{-0.15}^{+0.18}$ \\
\hline HCG 62 & & $37.70_{-0.22}^{+0.15}$ & $35.78_{-0.18}^{+0.22}$ \\
\hline M 84 & Sy 2 & $38.11_{-0.03}^{+0.03}$ & $35.04_{-0.20}^{+0.26}$ \\
\hline M 87 & $\mathrm{~L} 2$ & $39.30_{-0.10}^{+0.08}$ & $35.90_{-0.20}^{+0.27}$ \\
\hline M 89 & Sy 2 & $37.70_{-0.70}^{+0.26}$ & $34.48_{-0.18}^{+0.22}$ \\
\hline NGC 507 & & $38.30_{-0.30}^{+0.18}$ & $36.28_{-0.20}^{+0.24}$ \\
\hline NGC 1316 & & $37.70_{-0.70}^{+0.26}$ & $34.95_{-0.26}^{+0.28}$ \\
\hline NGC 4472 & Sy 2 & $37.95_{-0.18}^{+0.12}$ & $34.85_{-0.24}^{+0.23}$ \\
\hline NGC 4636 & $\mathrm{~L} 1$ & $36.70_{-0.10}^{+0.08}$ & $34.43_{-0.18}^{+0.19}$ \\
\hline NGC 5044 & & $37.30_{-0.30}^{+0.18}$ & $35.11_{-0.16}^{+0.21}$ \\
\hline NGC 5813 & L 2 & $37.00_{-0.22}^{+0.15}$ & $34.85_{-0.24}^{+0.27}$ \\
\hline NGC 5846 & L 2 & $37.11_{-0.64}^{+0.25}$ & $35.20_{-0.20}^{+0.24}$ \\
\hline
\end{tabular}

Notes: "Type" refers to optical AGN type; Sy 1/2: Seyfert 1/2 galaxies; L: LINERs; L 1/2: type 1/2 LINERs.

REFERENCES: Source types: NED; SIMBAD; Ho , Filippenko, \& Sargent (1997); Véron-Cetty \& Véron (2010). Powers: Balmaverde, Baldi, \& Capetti (2008); Russell et al. (2013); Nemmen \& Tchekhovskoy (2014).

\section{RESUlts AND Discussion}

Inspection of Figure 1 reveals that the sample galaxies follow a linear relation between $P_{\mathrm{B}}$ and $P_{\text {jet }}$ globally, albeit with substantial systematic scatter. As already noted by Allen et al. (2006), the presence of this relation, as well as the value found for $\eta$, indicate (i) that the accretion flows in the nuclei of radio galaxies are indeed well described by Bondi accretion in general, and (ii) that a significant fraction (about 1\%) of the restmass energy of the matter streaming into the Bondi radii of these systems ends up in jets.

Taking into account the optical AGN types, a remarkable pattern emerges: five out of six Seyfert 2 galaxies (the exception being UGC 6297) are located systematically below the best-fit linear relation between $P_{\mathrm{B}}$ and $P_{\text {jet }}$, by about $0.8 \mathrm{dex}$. Noting that most of the remaining galaxies are actually located above the model line, one finds that the five Sy 2 s are separate from the remainder of the sample by about one order of magnitude in terms of jet production efficiency. Even

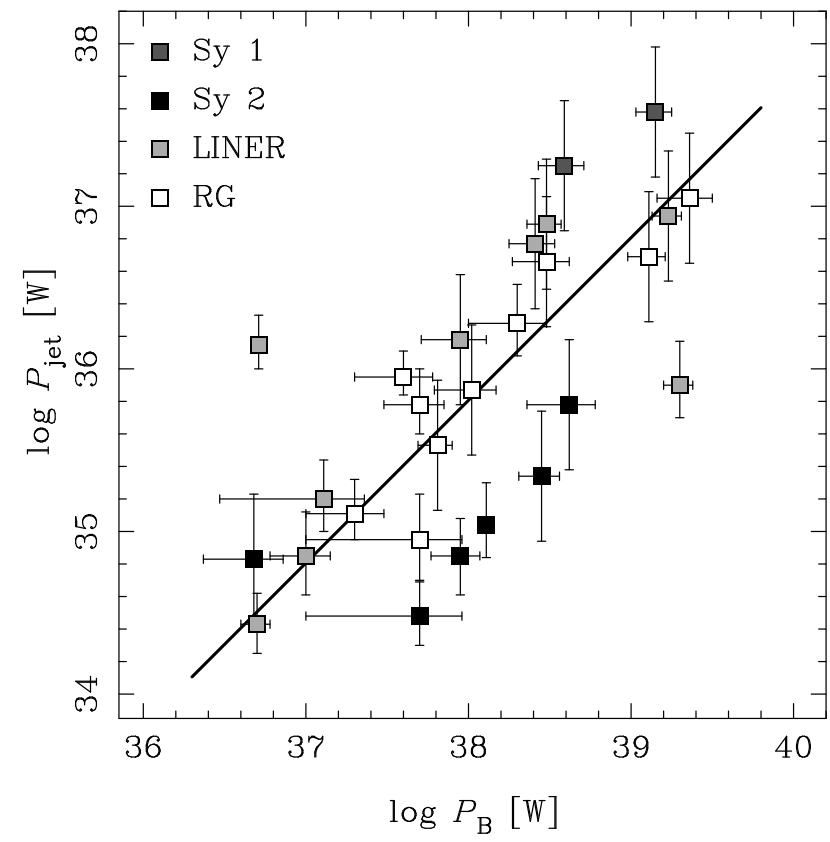

Figure 1. Kinetic jet power $P_{\text {jet }}$ as function of Bondi accretion power $P_{\mathrm{B}}$ using the data in Table 1. Optical AGN types are Seyfert 1 (Sy 1), Seyfert 2 (Sy 2), and LINER; RG denotes radio galaxies of unknown type. Error bars indicate $1 \sigma$ uncertainties. The diagonal continuous line corresponds to a linear relation with a jet production efficiency of $\eta \approx 0.6 \%$.

though selection effects cannot be ruled out entirely given the limited sample size, this distribution indicates that Seyfert 2 galaxies are physically different from the other sources. This discrepancy is statistically significant: there are six out of 27 galaxies located below the model line by more than their measurement errors in both $P_{\mathrm{B}}$ and $P_{\text {jet }}$, with five of them being Sy $2 \mathrm{~s}$. When drawing randomly six out of 27 sources, the probability of obtaining a set with at least five Sy $2 \mathrm{~s}$ is $4.3 \times 10^{-4}$, corresponding to a Gaussian significance of $3.5 \sigma$.

Within the frame of the standard viewing-angle unification scheme of AGN (Antonucci 1993; Urry \& Padovani 1995), the difference between type 1 (Sy 1 , LINER 1) and type 2 (Sy 2, LINER 2) galaxies arises from geometry: in case of type $2 \mathrm{AGN}$, the view into the central engine is obscured by a surrounding extended dust torus, whereas type 1 sources are seen at inclinations that permit a direct view into the central accretion zone. In contrast to this simple geometric explanation, Figure 1 suggests that the optical type of a given AGN is partially controlled by its jet production efficiency. A possible evolutionary description is the following: initially, Seyfert 2 galaxies are AGN which are relatively inefficient in powering jets and whose central regions are heavily obscured by dust. At some point, a sufficient - roughly one order of magnitude - increase in jet power, and thus in the release of energy in the central accretion zone, leads to two effects. Firstly, a partial dissolution of the surrounding dust torus. Depending on the viewing geometry, this may result in the transition of a Sy 2 to a type 1 galaxy; indeed, indications for 
such an evolutionary sequence were already reported by Tran (2003), Koulouridis et al. (2006) and, more recently, by Villarroel \& Korn (2014). Secondly, shockionization of the gas within the galactic nucleus by the jet. As already noted by Heckman (1980), this mechanism provides a natural explanation for the presence of the optical low-ionization emission lines characteristic for LINERs (see also Reynaldi \& Feinstein 2013; Couto et al. 2013; Riffel et al. 2014 for recent observational evidence for shock ionization by jets). Eventually, the scenario I propose implies an evolution from Seyfert $2 \mathrm{~s}$ to LINERs and Seyfert 1 galaxies caused by an increase in jet production efficiency. I note that the rather clear separation of the Seyfert 2 s from the rest of the sample suggests that the transition in efficiency happens relatively fast compared to the characteristic life times of galactic radio jets, meaning $\lesssim 10^{6}$ years (cf., e.g., Tadhunter et al. 2012).

When taking into account the apparent dependence of jet production efficiency on optical AGN type, the $P_{\mathrm{B}}-P_{\text {jet }}$ correlation could actually be much tighter than usually assumed. Indeed, when removing the six Sy 2 as well as the two outliers M 87 and NGC 4696 from the sample, I find an efficiency $\eta \approx 1 \%$ and an intrinsic scatter about the best-fit model line consistent with zero. A priori, some intrinsic scatter, as well as outliers, have to be expected because (i) the efficiency of accretion flows partially depends on the angular momentum of the infalling gas and the geometry and strength of the magnetic fields in the central accretion zone (e.g., Narayan \& Fabian 2011), (ii) AGN activity shows strong red-noise type temporal variability (e.g., Park \& Trippe 2012, 2014; Kim \& Trippe 2013), and (iii) a dependence of the AGN power on the physical properties of host galaxies, nearest-neighbor galaxies, and the large-scale background densities (Choi, Woo, \& Park 2009; Hwang et al. 2012). Those effects seem to be less influential in general than currently assumed once AGN evolution is taken into account properly.

\section{ACKNOWLEDGMEnTS}

This work made use of the NASA/IPAC Extragalactic Database, the SIMBAD Astronomical Database, and the software package DPUSER developed and maintained by Тномаs Отт at MPE Garching. I acknowledge financial support from the Korean National Research Foundation (NRF) via Basic Research Grant 2012-R1A1A2041387.

\section{REFERENCES}

Allen, S. W., et al. 2006, The Relation between Accretion Rate and Jet Power in X-ray Luminous Elliptical Galaxies, MNRAS, 372, 21

Antonucci, R. 1993, Unified Models for Active Galactic Nuclei and Quasars, ARA\&A, 31, 473

Balmaverde, B., Baldi, R. D., \& Capetti, A. 2008, The Accretion Mechanism in Low-Power Radio Galaxies, A\&A, 486, 119

Böttcher, M., Harris, D. E., \& Krawczynski, H. (eds.) 2012,
Relativistic Jets from Active Galactic Nuclei (Weinheim: Wiley-VCH)

Choi, Y.-Y., Woo, J.-H., \& Park, C. 2009, Environmental Dependence of Active Galactic Nucleus Activity. I. The Effects of Host Galaxy, ApJ, 699, 1679

Couto, G. S., et al. 2013, Kinematics and Excitation of the Nuclear Spiral in the Active Galaxy Arp 102B, MNRAS, 435,2982

Fujita, Y., Kawakatu, N., \& Shlosman, I. 2014, AGN Jet Power and Feedback Controlled by Bondi Accretion in Brightest Cluster Galaxies, arXiv:1406.6366

Heckman, T. M. 1980, An Optical and Radio Survey of the Nuclei of Bright Galaxies, A\&A, 87, 152

Ho, L. C. 2008, Nuclear Activity in Nearby Galaxies, ARA\&A, 46, 475

Ho, L. C., Filippenko, A. V., \& Sargent, W. L. 1997, A Search for "Dwarf" Seyfert Nuclei. III. Spectroscopic Parameters and Properties of the Host Galaxies, ApJS, 112, 315

Hwang, H. S., et al. 2012, Activity in Galactic Nuclei of Cluster and Field Galaxies in the Local Universe, A\&A, 538, A15

Kim, J.-Y., \& Trippe, S. 2013, How To Monitor AGN IntraDay Variability at $230 \mathrm{GHz}$, JKAS, 46, 65

King, A. L. 2013, Regulation of Black Hole Winds and Jets across the Mass Scale, ApJ, 762, 103

Koulouridis, E., et al. 2006, Local and Large-Scale Environment of Seyfert Galaxies, ApJ, 639, 37

Narayan, R., \& Fabian, A. C. 2011, Bondi Flow from a Slowly Rotating Hot Atmosphere, MNRAS, 415, 3721

Nemmen, R. S., \& Tchekhovskoy, A. 2014, On The Efficiency of Jet Production in Radio Galaxies, arXiv: 1406.7420

Netzer, H. 2013, The Physics and Evolution of Active Galactic Nuclei (Cambridge: Cambridge University Press)

Park, J.-H., \& Trippe, S. 2012, Multiple Emission States in Active Galactic Nuclei, JKAS, 45, 147

Park, J.-H., \& Trippe, S. 2014, Radio Variability and Random Walk Noise Properties of Four Blazars, ApJ, 785, 76

Reynaldi, V., \& Feinstein, C. 2013, Shock Ionization in the Extended Emission-Line Region of 3C 305: the Last Piece of the (Optical) Puzzle, MNRAS, 435, 1350

Riffel, R. A., et al. 2014, Feeding versus Feedback in NGC 1068 Probed with Gemini NIFS - I. Excitation, MNRAS, 442, 656

Russell, H. R., et al. 2013, Radiative Efficiency, Variability, and Bondi Accretion on to Massive Black Holes: the Transition from Radio AGN to Quasars in Brightest Cluster Galaxies, MNRAS, 432, 530

Tadhunter, C. N., et al. 2012, PKS 0347+05: a RadioLoud/Radio-Quiet Double Active Galactic Nucleus System Triggered in a Major Galaxy Merger, MNRAS, 427, 1603

Tran, H. D. 2003, The Unified Model and Evolution of Active Galaxies: Implications from a Spectropolarimetric Study, ApJ, 583, 632

Urry, M. C., \& Padovani, P. 1995, Unified Schemes for Radio-Loud Active Galactic Nuclei, PASP, 107, 803

Véron-Cetty, M.-P., \& Véron, P. 2010, A Catalogue of Quasars and Active Galactic Nuclei: 13th Edition, A\&A, 518, A10

Villarroel, B., \& Korn, A. 2014, The Different Neighbours around Type- 1 and Type-2 Active Galactic Nuclei, Nat. Phys., 10, 417 